\title{
ANÁLISIS DE LOS RESÚMENES DE ARTÍCULOS DE INVESTIGACIÓN DE DOS ÁREAS CIENTÍFICAS: UN ESTUDIO MULTIDISCIPLINARIO
}

\author{
Teresa Ramos-Quispe \\ Universidad Continental (Perú) \\ tramos@continental.edu.pe \\ Julio Efraín Postigo-Zumarán \\ Universidad Continental (Perú) \\ jpostigo@continental.edu.pe \\ Dennis Arias-Chávez \\ Universidad Continental (Perú) \\ darias@continental.edu.pe
}

Recibido: 19/08/2020 - Aprobado: 15/08/2020 - Publicado: 30/07/2021

DOI: doi.org/10.17533/udea.lyl.n80a19

\begin{abstract}
Resumen: Se busca describir la organización retórica de los resúmenes de artículos de investigación de seis revistas científicas peruanas adscritas a las ciencias biomédicas y sociales. Se analizó la estructura genérica de 270 textos publicados entre los años 2015 y 2019, con un enfoque descendente y bajo el modelo de movimientos retóricos (Swales \& Feak, 2009). Los resultados muestran la presencia de patrones de tres y cuatro movimientos en las ciencias sociales, y de cuatro movimientos en las biomédicas. Respecto al orden de aparición de los movimientos, las revistas de ciencias sociales presentan mayor variabilidad frente a las del área biomédica.

Palabras clave: resumen; movimientos retóricos; estructura retórica; ciencias biomédicas; ciencias sociales.

\section{RESEARCH ARTICLE'S ABSTRACTS ANALYSIS IN TWO SCIENTIFIC AREAS: A MULTIDISCIPLINARY STUDY}

\begin{abstract}
This article seeks to describe the rhetorical organization of the abstracts in research papers from six Peruvian scientific journals ascribed to the social and biomedical sciences. This research work analyzes the generic structure of 270 texts published between 2015 and 2019, with a descending approach and under the model of rhetorical movements (Swales $\&$ Feak, 2009). The results show the presence of three and four movements in the social sciences, and of four movements in the biomedical ones. Focusing on the movements' order of appearance, the social sciences journals show more variability than the biomedical journals.
\end{abstract}

Keywords: abstract; rhetorical movements; rhetorical structure; biomedical sciences; social sciences.

Editores

E-ISSN 2422-3174

N. ${ }^{\circ} 80,2021,319-339$

Juan Fernando Taborda Sánchez Juan Esteban Ibarra Atehortúa Victoria Gómez Peláez 
Análisis de los resúmenes de artículos de investigación de dos áreas científicas: un estudio multidisciplinario

\section{Introducción}

$\mathrm{L}$

os estudios sobre los géneros discursivos se han centrado en la vinculación de los intercambios verbales con la práctica social que se instaura en una sociedad, idea que se asocia a la perspectiva socio-comunicativa del género (Charaudeau, 2012). Otras perspectivas, como la sistémico-funcional, conciben los géneros como entidades complejas, conformadas por variables contextuales, semánticas y estructurales (Ciapuscio, 2005). Entrando en materia, el artículo de investigación — en adelante AI — es un género dinámico que no siempre responde a una estructura fija ni a un orden retórico establecido. Sus características suelen variar en el tiempo y su organización está determinada por las culturas académicas o científicas en las que está adscrito (Sánchez, 2016).

En la actualidad, la producción y circulación de material científico es tan amplia y tan variada que se hace complicado mantenerse al corriente de los nuevos conocimientos producidos. Esta situación conduce a la búsqueda de mecanismos que ayuden a acceder rápidamente al contenido de un AI antes de decidirse a leerlo por completo. Es así como surge el resumen, como una parte integrante del artículo de investigación (Swales, 1990) que presenta de manera condensada y precisa los contenidos de la investigación; si bien autores como Diez (2007) resaltan que en la redacción de este apartado, los autores suelen incluir información diversa sobre su estudio sin seguir un orden establecido, dando prioridad a determinados segmentos y dejando de lado otros, lo que puede restar potencia argumentativa y validez, dado que no permitiría tener un panorama completo de la investigación que presenta (Piqué-Noguera y Camaño-Puig, 2015).La estructura de los resúmenes debe reflejar las partes de los artículos de investigación — esto es resumen, introducción, método, resultados, discusión y conclusión- (Quintanilla, 2016). Esta relación se debe a que el resumen es el primer apartado con el que entrará en contacto el lector. Así, el estudio de cómo se configura el discurso académico en este apartado ayudará a conocer, en primer lugar, la información que contiene el AI sin necesidad de leer todo su contenido y, en segundo lugar, las convenciones existentes para la redacción de los resúmenes que se han establecido en los distintos ámbitos disciplinares.

\section{Movimiento retórico}

El movimiento retórico, llamado también movida retórica, es definido como la expresión de un propósito comunicativo en el cual cada uno de los fragmentos textuales en los que están subdivididas las secciones de un género discursivo comparten un mismo contenido semántico y una misma función comunicativa (Skelton, 1994). Este modelo propuesto por Swales $(1990,2004)$ está vinculado a la enseñanza de la escritura científica, sobre todo en inglés, y si bien la crítica que se hace de ella se vincula con su carácter descriptivo y prototípico, dado que no suele tomar en cuenta la variación disciplinar de los AI (Sabaj et al., 2011). Por ello, las herramientas de análisis que proporciona son perfectamente aprovechables en el ámbito de la escritura científica en español y en otras lenguas (Sabaj et al., 2011).Para Swales (1990), el resumen, como parte integrante del AI, ha sido uno de los apartados olvidados por los analistas del discurso. Si bien sus estudios — los de Swales — se centraron en analizar 
Análisis de los resúmenes de artículos de investigación de dos áreas científicas: un estudio multidisciplinario el apartado de la introducción del AI, con los años su propuesta se ha ido extendiendo a otros componentes como el resumen, el cual se amplió hasta formar un modelo mucho más integral basado en cuatro movimientos retóricos: I - Introducción_, M - Método_- R - Resultados - y D —Discusión — (Swales, 1990; Bhatia, 1993; Lorés, 2004). Tal modelo se ha visto enriquecido con la propuesta metadiscursiva de Hyland (2005), la cual se basa en el análisis de los recursos que utiliza el escritor para ayudar al lector a organizar y evaluar la información presentada (Beke, 2005).

\section{Antecedentes}

A partir de la propuesta de Swales (1990) se han desarrollado diversos estudios centrados en el análisis de los componentes del AI, con el fin de determinar los propósitos comunicativos y las estrategias retóricas seguidas para su elaboración. No son pocos los investigadores que, desde la perspectiva de los géneros discursivos, han utilizado el análisis de movimientos para describir la organización global de los resúmenes de artículos de investigación y las variaciones que este apartado presenta por áreas disciplinares (Samraj, 2005; Mur, 2007; Pho, 2008; Ibáñez \& Moncada, 2017). Una tendencia importante es el estudio del resumen desde un punto de vista interdisciplinario, en el que se comparan la estructura retórica, el metadiscurso y otras características lingüísticas de los resúmenes en diferentes disciplinas, con el fin de explorar sus variaciones.

En esta línea, autores como Alhuqbani (2013) y Martín (2003) han analizado el resumen en diversas disciplinas de las ciencias sociales; Can et al. (2016), Cárcamo (2020), Khansari et al. (2016), Salager-Meyer (2006), Tseng (2011) y Suntara y Usaha (2013) se han centrado en analizar la estructura de resúmenes de artículos de lingüística aplicada, lingüística y medicina; también se destaca el estudio de Cignetti (2017), que hace lo mismo en resúmenes de medicina veterinaria; El-Dakhs (2018) y su estudio comparativo de los resúmenes en revistas de alto y bajo impacto; Hartley $(1997,2002)$ con su estudio sobre las características y tipos de resúmenes de artículos de investigación; y, finalmente, Quintanilla y Kloss (2019) con su estudio comparativo de los resúmenes en artículos de investigación de ciencias sociales y ciencias básicas.

\section{Objetivo del estudio}

La presencia de trabajos sobre análisis interdisciplinario de resúmenes de AI en revistas científicas son diversos; no obstante, no se han encontrado trabajos que, por un lado, se centren en analizar las características retorico-discursivas de este apartado en revistas científicas peruanas y, por otro lado, comparen los resúmenes para hallar diferencias y puntos en común que expliquen su regularidad y organización. Tales vacíos motivaron a los autores a emprender el presente estudio.

El objetivo de este trabajo, entonces, es describir la organización retórica de los resúmenes de artículos de investigación de seis revistas científicas peruanas adscritas a las áreas de las ciencias biomédicas y de las 
Análisis de los resúmenes de artículos de investigación de dos áreas científicas: un estudio multidisciplinario ciencias sociales. Los hallazgos permitirán caracterizar por áreas disciplinares los artículos publicados en el Perú y ayudarán a develar la retórica de esas disciplinas.

\section{Descripción del corpus y método de análisis}

\subsection{Selección del corpus y estadística básica}

El corpus está compuesto por 270 resúmenes de artículos de investigación originales, extraídos de seis revistas peruanas de dos áreas disciplinares: ciencias sociales (con 120 resúmenes de tipo tradicional) y ciencias biomédicas (con 150 resúmenes de tipo estructurado), publicados entre 2015 y 2019 — ver la Tabla 1—. Las revistas seleccionadas pertenecen a las disciplinas de lingüística, comunicación y psicología para el caso de las ciencias sociales; mientras que para las ciencias biomédicas se seleccionaron dos revistas de medicina humana y una de medicina veterinaria. Estas revistas fueron seleccionadas por ser las publicaciones más representativas de su área en el Perú y por estar indexadas en bases de datos importantes, como Scopus y SciELO.

\begin{tabular}{|c|c|c|c|c|c|c|c|}
\hline \multirow{2}{*}{ Áreas } & Disciplinas & $\mathbf{2 0 1 5}$ & $\mathbf{2 0 1 6}$ & $\mathbf{2 0 1 7}$ & $\mathbf{2 0 1 8}$ & $\mathbf{2 0 1 9}$ & Total \\
\hline \multirow{3}{*}{$\begin{array}{c}\text { Ciencias } \\
\text { sociales }\end{array}$} & Comunicación & 5 & 5 & 10 & 10 & 10 & 40 \\
\cline { 2 - 8 } & Psicología & 10 & 9 & 10 & 10 & 10 & 49 \\
\cline { 2 - 8 } & Lingüística & 3 & 5 & 7 & 5 & 11 & 31 \\
\hline \multirow{4}{*}{$\begin{array}{c}\text { Ciencias } \\
\text { biomédicas }\end{array}$} & Medicina humana I & 10 & 10 & 10 & 10 & 10 & 50 \\
\cline { 2 - 8 } & Medicina veterinaria & 10 & 10 & 10 & 10 & 10 & 50 \\
\cline { 2 - 8 } & Medicina humana II & 10 & 10 & 10 & 10 & 10 & 50 \\
\hline
\end{tabular}

Tabla 1. Relación de áreas, disciplinas y números de revistas evaluados por año

El siguiente paso fue identificar los movimientos o elementos discursivos de los resúmenes. Una vez completada esta etapa, se procedió a realizar cálculos simples de frecuencia. Este análisis se realizó teniendo en cuenta la función o contenido del texto, utilizando un enfoque descendente o top-down. Al haberse seleccionado el corpus, se etiquetó cada una de las unidades de análisis y se realizó el conteo de palabras por resumen y se calculó su desviación estándar. En la Tabla 2 se presentan los datos numéricos básicos correspondientes a las seis revistas analizadas. 


\begin{tabular}{|c|c|c|c|c|}
\hline Área & Artículo & $\begin{array}{c}\text { Cantidad de } \\
\text { palabras }\end{array}$ & $\begin{array}{c}\text { Palabras por } \\
\text { resumen }\end{array}$ & $\begin{array}{c}\text { Desviación } \\
\text { estándar }\end{array}$ \\
\hline \multirow{4}{*}{$\begin{array}{c}\text { Ciencias } \\
\text { sociales }\end{array}$} & Comunicación & 4933 & 123,33 & 28,61 \\
\cline { 2 - 5 } & Psicología & 6491 & 132,47 & 26,35 \\
\cline { 2 - 5 } & Lingüística & 3969 & 128,03 & 31,24 \\
\hline \multirow{4}{*}{$\begin{array}{c}\text { Ciencias } \\
\text { biomédicas }\end{array}$} & Medicina humana I & 11162 & 223,24 & 38,57 \\
\cline { 2 - 5 } & Medicina veterinaria & 8598 & 171,96 & 55,47 \\
\cline { 2 - 5 } & Medicina humana II & 11530 & 230,60 & 32,12 \\
\hline
\end{tabular}

Tabla 2. Relación entre la cantidad de palabras, las palabras por resumen y la desviación estándar en las revistas seleccionadas, donde la desviación estándar indica qué tan dispersos están los datos con respecto a la media

En la Tabla 2 se puede observar una alta desviación estándar en las revistas analizadas, lo cual evidencia una alta variabilidad en la extensión de los resúmenes. Tal es el caso de la revista de medicina veterinaria, con un mínimo y máximo de palabras por resumen de 52 y 258, con lo cual la desviación se eleva a 55,47 \%. Lo mismo sucede con la revista de medicina humana I, con 130 y 311 palabras y una desviación del 38,57 \%. Para el caso de las revistas del área de ciencias sociales, la desviación no es tan alta como ocurre en las de ciencias biomédicas. El caso más resaltante es el de la revista de lingüística, con 72 y 198 palabras y una desviación del 31,24 \%. La revista de psicología, por su parte, es la que presenta la menor desviación estándar en relación con las demás, aunque no por ello sea menos significativa a nivel global, dado que el mínimo de palabras hallado es de 102 y el máximo de 259. Un dato importante que determina el porcentaje de desviación hallada es el número total de palabras, el cual es mayor en las revistas del área de ciencias biomédicas —31 290 en total— que en las revistas de ciencias sociales - 15393 en total-.

\section{Método de análisis}

Para el análisis de las unidades de estudio se siguió el modelo propuesto por Swales y Feak (2009), move y step o «movimiento» y «paso» — ver la Tabla 3-, con el fin de analizar y clasificar los movimientos retóricos presentes en los resúmenes de los AI de las revistas seleccionadas. A diferencia de otros modelos, como los de Weissberg y Burker (1990), Dos Santos (1996) y Hyland (2000), que plantean una estructura basada en cinco movidas debidamente etiquetadas, el modelo de Swales y Feak se caracteriza por proponer cinco movimientos asociados a preguntas «implícitas» que deben ser respondidas por el investigador para redactar cada uno de los apartados del resumen, lo cual le brinda una ventaja didáctica frente a las demás. 
Análisis de los resúmenes de artículos de investigación de dos áreas científicas: un estudio multidisciplinario

\begin{tabular}{c|c|c}
\hline Movidas & Etiquetas típicas & Preguntas implicadas \\
\hline Movimiento 1 & Antecedentes/Introducción/Situación & ¿Qué sabemos acerca del tema? \\
\hline Movimiento 2 & Presentar investigación/propósito & ¿De qué trata el estudio? \\
\hline Movimiento 3 & Métodos/Materiales/Sujetos/Procedimientos & ¿Cómo fue hecho? \\
\hline Movimiento 4 & Resultados/Hallazgos & ¿Qué fue descubierto? \\
\hline Movimiento 5 & Discusión/Conclusión/Implicación/Aplicaciones & ¿Qué significan los hallazgos?
\end{tabular}

Tabla 3. Clasificación de movidas retóricas propuesta por Swales y Feak (2009)

\subsection{Análisis cualitativo}

Se recurrió al análisis manual de cada uno de los resúmenes para conocer la intención comunicativa de los autores, identificable en el uso de ciertas estrategias retóricas. Los apartados objeto de análisis desarrollan una retórica particular que permite reconocer el propósito que tiene en mente el autor. Para ilustrar este hecho, se tiene que en el movimiento 1 - ¿Qué sabemos acerca del tema?»- el autor pone el objeto de estudio en contexto y expone los antecedentes o rasgos identificatorios del fenómeno. Desde un punto de vista gramatical, en el ejemplo (1) se puede observar que el autor recurre a oraciones yuxtapuestas con verbo en tiempo presente para reforzar su idea; mientras que en (2) el autor emplea oraciones yuxtapuestas, en tiempo presente la primera y pretérito perfecto compuesto la segunda.

(1) El uso de Twitter con fines de comunicación externa se ha generalizado entre las instituciones de educación universitaria. Los mensajes breves y el libre acceso a la información han convertido a Twitter en un canal idóneo para gestionar y controlar la comunicación reputacional en las instituciones (Atarama \& Cortez, 2015).

(2) El yeísmo, así como su rehilamiento y ensordecimiento, son rasgos caracterizadores del español del Uruguay. Varios estudios locales han indagado sobre estos fenómenos atendiendo tanto a cuestiones diacrónicas como sincrónicas (Canale \& Coll, 2016).

Como la exposición de la finalidad o propósito del trabajo es la razón de ser del movimiento 2 — «De qué trata el estudio?»—, este, por lo general, se escribe de modo más claro, lo que se evidencia por el empleo de términos como «objetivo», «propósito» o «finalidad», según se observa a continuación:

(3) La presente investigación tuvo como objetivo analizar y describir las prácticas o conductas de salud que presentan un grupo de estudiantes en sus primeros años de estudio de una universidad privada de Lima (Becerra, 2016).

Con todo, es frecuente encontrar al movimiento 2 cumpliendo la función de describir las acciones o actividades que el autor emprendió en el trabajo de investigación, con lo cual el objetivo de la investigación queda claro: 
(4) Este estudio examina las relaciones entre la identificación colectiva con una comunidad rural y las expresiones subjetiva, psicológica y social del bienestar (Espinosa et al., 2016).

En el área de las ciencias biomédicas se suelen estructurar los resúmenes recurriendo a marcas o palabras que demarcan los apartados del artículo, lo cual es característico de los resúmenes de tipo estructurado, los cuales abarcan la misma información que los resúmenes de otro tipo pero añaden etiquetas a los apartados con el fin de facilitar la transmisión de la información (Altman \& Gardner, 1987; Hartley, 1997, 2002; SalagerMeyer, 2006; Arias-Chávez et al., 2019). La revista de medicina humana I y medicina humana II siguen este modelo de resumen. A continuación, un ejemplo de la manera en la que los artículos de estas revistas presentan el movimiento 3 — «Cómo fue hecho?»—, cuyo objetivo es presentar de forma clara los procedimientos — tipo, diseño, población, muestra, entre otros - llevados a cabo para la realización del estudio:

(5) Material y métodos: La muestra estuvo constituida por 152 estudiantes entre 12 y 17 años con dentición permanente. Se tomaron registros fotográficos en norma frontal con los estudiantes en posición natural de la cabeza. Sobre las fotografías impresas se determinó el ángulo de apertura facial para establecer el biotipo facial. El registro de la sobremordida vertical se realizó de forma clínica con la ayuda de un calibrador Vernier y un lápiz dermatográfico (Sánchez-Tito et al., 2015).

(6) Materiales y métodos: Estudio de casos-controles pareados. Se definió como caso al niño que no completó 11 controles CRED hasta cumplir 11 meses de edad, y como control, aquel niño con 11 controles CRED para la misma edad. La relación entre cada factor con el cumplimiento del CRED se estableció mediante la prueba de McNemar $(p<0,05)$ y como medida de fuerza de asociación el odds ratio (OR) para muestras pareadas (IC $95 \%$ ) (GonzalesAchuy et al., 2016).

Por otro lado, en las revistas de ciencias sociales suele producirse una integración de este apartado en el texto, esto es, no se recurren a marcas que la destaquen entre los demás apartados, así:

(7) Este es un estudio mixto-secuencial explicativo. En la primera fase se clasifica a los participantes utilizando un análisis de correspondencia múltiple; y en la segunda y fase principal, se analizan tres entrevistas diferentes realizadas a nueve maestros (García-Cepero et al., 2016).

El movimiento 4 «¿Qué fue descubierto?», que cumple el cometido de presentar los principales resultados producto de la investigación, queda ilustrado en el ejemplo (8). Con frecuencia se encuentra este movimiento señalado con términos como «los resultados evidencian, muestran, aportan...». Este movimiento puede incluirse, como se ve en el caso (9), en resúmenes de tipo estructurado, en el que no aparece la expresión «Los resultados».

(8) Los resultados de dicho análisis aportan datos sobre la construcción y la relación entre diferentes identidades, y la discusión de los resultados gira en torno a un núcleo principal: el poder (Donstrup, 2018). 
(9) Resultados. Se identificaron 40 pares de casos y controles. Los casos estuvieron hospitalizados más de dos semanas y emplearon más de dos antibióticos. Los costos directos sanitarios asociados fueron por hospitalización, antibióticos, exámenes auxiliares, evaluaciones especializadas y otras medicaciones (Dámaso-Mata et al., 2016).

El movimiento 5 - ¿Qué significan los hallazgos?»— sirve para analizar los hallazgos y se destacan sus consecuencias y aplicaciones. Entre los términos usados más frecuentemente se tienen, entre otros: «se sugiere», «se muestra», «se concluye»y «se establece». Es pertinente añadir que la frase «se muestra» puede presentarse también en la estructura del movimiento 4, según se ve en (11):

(10) En este estudio, se concluve que el aimara de Conima forma compuestos a partir de seis patrones sintácticos que siguen la estructura complemento-núcleo. Finalmente, en relación con su tipología, en la indagación se indica que esta variedad emplea la composición para formar palabras compuestas básicamente de orden nominal (Lovón, 2019).

(11) Desde un punto de vista formal, este estudio muestra la flexibilidad sintáctica de la estructura, en la cual es frecuente encontrar interpolaciones, elementos enfatizadores como pero e incluso dobles focalizaciones (Diez del Corral, 2018).

Cabe señalar que al final del resumen también pueden insertarse propuestas o recomendaciones. Esta información se suele incluir en la estructura del movimiento 5 —¿Qué significan los hallazgos?»—, según se observa en (12):

(12) Proponemos que la afectación es un operador ubicado en una proyección externa a Sv, el cual cuantiza el evento determinando la especificidad del estado resultante para el objeto; y la forma morfológica del operador es a- o en- en caso de que su valor sea positivo o negativo, respectivamente (Martínez, 2016).

\section{Análisis de los resultados}

\subsection{Movimientos retóricos}

En el presente apartado se muestran los resultados obtenidos tras la identificación de los movimientos retóricos de los resúmenes analizados de las seis revistas.

En la Tabla 4 se puede observar que la revista de psicología aparece como la más completa, con un porcentaje promedio de movimientos de 72,2 \%. Este resultado es similar a lo hallado por Martín (2003), quien obtuvo un cómputo global de 73,75 \% en resúmenes de psicología experimental, aunque inferior a lo hallado por Hyland (2000), quien determinó un porcentaje global de movidas de 58,8 \%. Le sigue la revista de comunicación, con un promedio de movidas de 63,8 \%; y la de lingüística, con un 63,2 \%. Respecto a la presencia de los movimientos, se puede observar que el M1 — ¿Qué sabemos acerca del tema?»-y el M5 — ¿Qué significan los hallazgos?»— presentan en promedio porcentajes menores — 19,3\% y 16,7 \%, respectivamente— en comparación con los otros 
Análisis de los resúmenes de artículos de investigación de dos áreas científicas: un estudio multidisciplinario movimientos. A partir de estos resultados, es posible concluir que los movimientos 2, 3 y 4 son obligatorios en las tres disciplinas en las que se encuentran enmarcadas las revistas estudiadas, en tanto que el M1 es opcional, sobre todo para el caso de la revista de lingüística, resultado que coincide con lo hallado por Alhuqbani (2013).

\begin{tabular}{|c|c|c|c|c|c|c|c|c|c|c|c|c|}
\hline Revista & M1 & M1 \% & M2 & M2\% & M3 & M3 \% & M4 & M4 \% & M5 & M5 \% & \multicolumn{2}{|c|}{$\begin{array}{l}\text { Promedio } \\
\text { M } \%\end{array}$} \\
\hline Comunicación & 29 & 73 & 37 & 93 & 30 & 75 & 18 & 45 & 13 & 33 & 25.4 & 63.8 \\
\hline Psicología & 12 & 24 & 47 & 96 & 47 & 96 & 46 & 94 & 25 & 51 & 35.4 & 72.2 \\
\hline Lingüística & 17 & 55 & 27 & 87 & 24 & 77 & 18 & 58 & 12 & 39 & 19.6 & 63.2 \\
\hline Total & 58 & 48 & 111 & 93 & 101 & 84 & 82 & 68 & 50 & 42 & & \\
\hline Promedio & 19,3 & - & 37,0 & - & 33,7 & - & 27,3 & - & 16,7 & - & & \\
\hline
\end{tabular}

Tabla 4. Resultados porcentuales de las movidas retóricas en las revistas seleccionadas del área de ciencias sociales

Para el caso de las revistas de ciencias biomédicas — ver la Tabla 5-, la revista de medicina humana I es la que presenta un promedio de $82 \%$ de movimientos, a excepción del M1 — ¿Qué sabemos acerca del tema?»Le sigue la revista de medicina humana II, en la cual ocurren cuatro movimientos, con excepción del M1; y la revista de medicina veterinaria, con un $70 \%$. En líneas generales, los movimientos 2, 3, 4 y 5 son obligatorios en las tres revistas analizadas. No obstante, el M1 tiene una presencia menor en las revistas de medicina humana I y medicina veterinaria, y ninguna en la revista de medicina humana II.

\begin{tabular}{|c|c|c|c|c|c|c|c|c|c|c|c|}
\hline Revista & M1 & M1 \% & M2 & М2 \% & M3 & M3 \% & M4 & M4\% & M5 & $\begin{array}{c}\text { M5 } \\
\%\end{array}$ & $\begin{array}{c}\text { Promedio } \\
\text { M } \%\end{array}$ \\
\hline Medicina humana I & 5 & 10 & 50 & 100 & 50 & 100 & 50 & 100 & 50 & 100 & $41 \quad 82$ \\
\hline Medicina veterinaria & 7 & 14 & 48 & 96 & 46 & 92 & 46 & 92 & 28 & 56 & 35 \\
\hline Medicina humana II & 0 & 0 & 50 & 100 & 50 & 100 & 50 & 100 & 50 & 100 & 40 \\
\hline Total & 12 & 8 & 148 & 99 & 146 & 97 & 146 & 97 & 128 & 851 & - \\
\hline Promedio & 3,5 & - & 49,0 & - & 48,0 & - & 48,0 & - & 39,0 & & - \\
\hline
\end{tabular}

Tabla 5. Resultados porcentuales de las movidas retóricas en las revistas seleccionadas del área de ciencias biomédicas

Sobre la cantidad de movimientos en un resumen - Tabla 6-, autores como Weissberg y Burker (1990) consideran que lo ideal es que haya presencia de los cinco movimientos. Siguiendo esta lógica, en los 270 resúmenes analizados deben figurar 1350 movimientos: 750 en los 150 resúmenes de las revistas del área de 
Análisis de los resúmenes de artículos de investigación de dos áreas científicas: un estudio multidisciplinario ciencias biomédicas y 600 en los 120 resúmenes de las revistas del área de ciencias sociales. No obstante, este hecho no se logra comprobar. Los movimientos con mayor presencia en ambas áreas son M2 — «De qué trata el estudio?»-, M3 — ¿Cómo fue hecho?»-, M4 — ¿Qué fue descubierto?»— y M5 — ¿Qué significan los hallazgos?»—, en tanto que el M1 — ¿Qué sabemos acerca del tema?»— se ubica en el último lugar de presencia en los resúmenes, con un 14,10\% en las revistas de ciencias sociales y 2,07 \% en las de ciencias biomédicas.

\begin{tabular}{|c|c|c|c|c|c|}
\hline \multicolumn{3}{|c|}{ Ciencias sociales } & \multicolumn{3}{c|}{ Ciencias biomédicas } \\
\hline Movimiento & Fi & \% & Movimiento & Fi & \% \\
\hline M1 & 58 & 14,10 & M1 & 12 & 2,07 \\
\hline M2 & 111 & 27,10 & M2 & 148 & 25,52 \\
\hline M3 & 101 & 24,60 & M3 & 146 & 25,17 \\
\hline M4 & 82 & 20,00 & M4 & 146 & 25,17 \\
\hline M5 & 58 & 14,10 & M5 & 128 & 22,07 \\
\hline $\begin{array}{c}\text { De 120 } \\
\text { resúmenes }\end{array}$ & $\mathbf{4 1 0}$ & $\mathbf{1 0 0 , 0 0}$ & $\begin{array}{c}\text { De 150 } \\
\text { resúmenes }\end{array}$ & $\mathbf{5 8 0}$ & $\mathbf{1 0 0 , 0 0}$ \\
\hline
\end{tabular}

Tabla 6. Relación porcentual de la presencia de los movimientos retóricos en los resúmenes según el área de las revistas seleccionadas

Respecto a la frecuencia de aparición de los movimientos, los resultados coinciden con el estudio de Cross y Openheim (2005), quienes asignan a los movimientos 2, 3 y 4 un porcentaje promedio de $80 \%$ de presencia en los artículos científicos. También coinciden con lo obtenido por Quintanilla y Kloss (2019), quienes determinaron que los movimientos más frecuentes en los resúmenes de artículos de lingüística, educación y psicología corresponden a los movimientos 2 y 3 , con al menos un $80 \%$ de ocurrencia; en tanto que el movimiento 4 es más frecuente en la disciplina de la psicología, con un $95 \%$ de recurrencia.

Finalmente, en lo que concierne al movimiento 5 —¿Qué significan los hallazgos?»—, se tiene mayor presencia en las revistas del área de las ciencias biomédicas que en las de ciencias sociales con porcentajes de 14 $\%$ y $22,07 \%$, respectivamente. Tal promedio es mayor al del movimiento 1 «¿Qué sabemos acerca del tema?, el cual se presenta solo en un 2,07\% en las revistas de ciencias biomédicas, frente a un 14,10\% en las de ciencias sociales. Los resultados coinciden con los obtenidos por Hyland (2000), quien, en un estudio multidisciplinar de resúmenes de investigación, determinó que existe una tendencia entre los autores de las ciencias positivas a priorizar la descripción de sus métodos y los resultados de sus estudios en detrimento de la contextualización de su trabajo mediante la introducción. Los autores de las ciencias sociales, continúa Hyland, buscan diversas estrategias retóricas para familiarizar a los lectores con su tema de estudio, de allí una mayor presencia del movimiento 1 . 


\subsection{Estructura u orden de los movimientos}

Al analizar el orden de los movimientos en cada uno de los resúmenes estudiados en las revistas del área de ciencias sociales — ver la Tabla 7—, se ve que existe una alta presencia de repeticiones y alteraciones en el orden de los movimientos, como en los siguientes patrones: M1 M2 M1 M2 M5, de la revista de comunicación; M2 M3 M2 M4 M5, de la revista de psicología; y M2 M3 M4 M1 M5 M4, de la revista de lingüística.

Ahora bien, la revista de comunicación presenta la mayor cantidad de patrones para organizar los componentes del resumen — 23 en total—, donde el patrón de cuatro movimientos M1 M2 M3 M4 es el más frecuente, con un $14,63 \%$ - aunque en el global prevalece el patrón de tres movimientos_. Por su parte, la revista de psicología _con 13 patrones — destaca el patrón de tres movimientos M2 M3 M4, con un 32,65 \% de frecuencia y de cuatro movimientos M2 M3 M4 M5, con un 30,61\% de ocurrencia.

Finalmente, la revista de lingüística ofrece 21 patrones diferentes. Ahora bien, pese a que el patrón con dos movimientos M2 M3 es el que se presenta más frecuentemente con un 16,13\%, es importante recalcar que, en general, predominan los resúmenes organizados en tres y cuatro movimientos. Este resultado coincide parcialmente con lo hallado por Suntara y Usaha (2013), Can et al. (2016) y El-Dakhs (2018) en sus estudios sobre resúmenes en artículos de lingüística y lingüística aplicada, en los que se determinó que la estructura recurrente de los resúmenes en estas disciplinas es de cuatro movimientos, similar a la estructura del artículo de investigación, esto es, introducción, método, resultados y discusión (Khansari et al., 2016; Tseng, 2011).

\begin{tabular}{|c|c|c|}
\hline \multicolumn{3}{|c|}{ Comunicación } \\
\hline Estructura & Fi & \% \\
\hline M1 & 1 & 2,44 \\
\hline M1 M2 & 3 & 7,32 \\
\hline M1 M2 M1 M2 M5 & 1 & 2,44 \\
\hline M1 M2 M1 M3 & 1 & 2,44 \\
\hline M1 M2 M3 & 3 & 7,32 \\
\hline M1 M2 M3 M4 & 6 & 14,63 \\
\hline M1 M2 M3 M5 & 4 & 9,76 \\
\hline M1 M2 M4 & 1 & 2,44 \\
\hline M1 M2 M5 & 1 & 2,44 \\
\hline M1 M3 M2 M3 M4 & 1 & 2,44 \\
\hline M1 M3 M4 & 1 & 2,44 \\
\hline M2 M1 & 2 & 4,88 \\
\hline M2 M1 M3 & 1 & 2,44 \\
\hline M2 M1 M3 M4 M5 & 1 & 2,44 \\
\hline M2 M1 M4 M5 & 1 & 2,44 \\
\hline M2 M3 & 1 & 2,44 \\
\hline
\end{tabular}


Análisis de los resúmenes de artículos de investigación de dos áreas científicas: un estudio multidisciplinario

\begin{tabular}{|c|c|c|}
\hline M2 M3 M1 & 1 & 2,44 \\
\hline M2 M3 M4 & 3 & 7,32 \\
\hline M2 M3 M4 M5 & 3 & 7,32 \\
\hline M2 M3 M5 & 1 & 2,44 \\
\hline M2 M5 & 1 & 4,88 \\
\hline M3 M2 & 2 & 4,88 \\
\hline Total: $\mathbf{2 3}$ & $\mathbf{4 0}$ & $\mathbf{1 0 0}$ \\
\hline
\end{tabular}

\begin{tabular}{|c|c|c|}
\hline \multicolumn{3}{|c|}{ Psicología } \\
\hline Estructura & Fi & $\mathbf{\%}$ \\
\hline M2M1M3M4M5 & 1 & 2,04 \\
\hline M1M2M3M4 & 3 & 6,12 \\
\hline M1M2M3M4M5 & 3 & 6,12 \\
\hline M1M2M4 & 1 & 2,04 \\
\hline M1M3M4 & 1 & 2,04 \\
\hline M1M3M4M5 & 1 & 2,04 \\
\hline M2M1M3M4 & 2 & 4,08 \\
\hline M2M3M2M4M5 & 1 & 2,04 \\
\hline M2M3M4 & 16 & 32,65 \\
\hline M2M3M4M3M4M5 & 1 & 2,04 \\
\hline M2M3M4M5 & 15 & 30,61 \\
\hline M2M3M5 & 3 & 6,12 \\
\hline M2M4 & 1 & 2,04 \\
\hline Total: 13 & $\mathbf{4 9}$ & $\mathbf{1 0 0}$ \\
\hline
\end{tabular}

\begin{tabular}{|c|c|c|}
\hline \multicolumn{3}{|c|}{ Lingüística } \\
\hline Estructura & $\mathbf{F i}$ & $\mathbf{\%}$ \\
\hline M1 M4 & 2 & 6,45 \\
\hline M1 M2 & 1 & 3,23 \\
\hline M1 M2 M3 M4 M2 & 1 & 3,23 \\
\hline M1 M2 M3 M4 M5 & 1 & 3,23 \\
\hline M1 M2 M3 M5 & 1 & 3,23 \\
\hline M1 M5 & 1 & 3,23 \\
\hline M2 M1 M3 & 3 & 9,68 \\
\hline M2 M1 M3 M4 M5 & 1 & 3,23 \\
\hline M2 M1 M4 M3 & 1 & 3,23 \\
\hline M2 M1 M5 M3 & 1 & 3,23 \\
\hline M2 M3 & 5 & 16,13 \\
\hline M2 M3 M1 M4 M5 & 1 & 3,23 \\
\hline M2 M3 M2 M4 M5 & 1 & 3,23 \\
\hline M2 M3 M4 & 4 & 12,90 \\
\hline M2 M3 M4 M1 M5 M4 & 1 & 3,23 \\
\hline
\end{tabular}




\begin{tabular}{|c|c|c|}
\hline M2 M3 M4 M5 & 1 & 3,23 \\
\hline M2 M4 & 1 & 3,23 \\
\hline M2 M4 M5 & 1 & 3,23 \\
\hline M3 M1 M2 M4 & 1 & 3,23 \\
\hline M3 M1 M2 M5 & 1 & 3,23 \\
\hline M5 M2 M5 & 1 & 3,23 \\
\hline Total: 21 & $\mathbf{3 1}$ & $\mathbf{1 0 0}$ \\
\hline
\end{tabular}

Tabla 7. Relación de los patrones retóricos presentes en los resúmenes de las revistas seleccionadas del área de ciencias sociales

En relación con los patrones de los resúmenes en el área de las ciencias biomédicas, estas presentan una baja variabilidad, con tan solo 15 patrones diferentes en comparación con los 57 que presentan los resúmenes del área de ciencias sociales. La revista de medicina veterinaria, por su parte, concentra la mayor cantidad de patrones - 12 - organizados en tres y cuatro movimientos - $30 \%$ y $40 \%$, respectivamente- Este resultado coincide parcialmente con lo hallado por Cignetti y Guzmán (2017), quienes determinaron que en la mayoría de los resúmenes de artículos analizados de dicha área en inglés prevalecen los patrones basados en cuatro movimientos. En lo que respecta a la revista de medicina humana i y revista de medicina humana II, la presencia de patrones de cuatro movimientos supera el $90 \%$, lo que coincide con el estudio de Cárcamo (2020) y sus resultados sobre la variación de la organización retórica en la disciplina de medicina. En la Tabla 8 se puede observar que en las revistas de medicina humana I y il no existen alteraciones en el orden de los movimientos, fenómeno que sí se presenta en la revista de medicina veterinaria — véanse los patrones M1 M3 M5 M3 M2 y M2 M4 M3 M4-. 


\begin{tabular}{|c|c|c|c|}
\hline Área & Estructura & $\mathbf{F i}$ & $\mathbf{\%}$ \\
\hline \multirow{3}{*}{$\begin{array}{c}\text { Medicina } \\
\text { humana I }\end{array}$} & M1M2M3M4M5 & 4 & 8 \\
\cline { 2 - 4 } & M2M3M4M5 & 46 & 92 \\
\cline { 2 - 4 } & Total: 2 & $\mathbf{5 0}$ & $\mathbf{1 0 0}$ \\
\hline \multirow{4}{*}{$\begin{array}{c}\text { Medicina } \\
\text { veterinaria }\end{array}$} & M1M2 & 2 & 4 \\
\cline { 2 - 4 } & M1M2M3M4 & 2 & 4 \\
\cline { 2 - 4 } & M1M2M3M4M5 & 2 & 4 \\
\cline { 2 - 4 } & M1M3M5M3M2 & 1 & 2 \\
\cline { 2 - 4 } & M2M3M4 & 15 & 30 \\
\cline { 2 - 4 } & M2M3M5 & 22 & 44 \\
\cline { 2 - 4 } & M2M4 & 1 & 2 \\
\cline { 2 - 4 } & M2M4M3M4 & 1 & 2 \\
\cline { 2 - 4 } & M2M4M5 & 1 & 2 \\
\cline { 2 - 4 } & M3M4 & 1 & 2 \\
\cline { 2 - 4 } & M3M4M5 & 1 & 2 \\
\cline { 2 - 4 } & Total: 12 & $\mathbf{5 0}$ & $\mathbf{1 0 0}$ \\
\hline \multirow{7}{*}{$\begin{array}{c}\text { Medicina } \\
\text { humana II }\end{array}$} & M2M3M4M5 & 50 & 100 \\
\cline { 2 - 4 } & Total: 1 & $\mathbf{5 0}$ & $\mathbf{1 0 0}$ \\
\hline
\end{tabular}

Tabla 8. Relación porcentual de los patrones retóricos presentes en los resúmenes de las revistas seleccionadas del área de ciencias biomédicas

\subsection{Movimientos realizados}

El número de movimientos que contienen los resúmenes de los artículos se analizan en esta sección. Los datos de la Tabla 9 muestran que, para el caso de las revistas del área de ciencias biomédicas, solo en 7 de los 150 resúmenes ocurren los cinco movimientos, mientras que para el caso de las revistas del área de ciencias sociales, 15 de 120 resúmenes presentan los cinco movimientos. No obstante, la presencia de cuatro movimientos es mayor en las revistas del área biomédica — 80,7 \% - que en las de ciencias sociales, en las que la presencia de resúmenes con tres y cuatro movimientos comparte el mismo porcentaje. Estos resultados coinciden en parte con los hallazgos de Hartley y Betts (2009), quienes señalan que, para el caso de las ciencias sociales, los resúmenes estructurados con los movimientos M2, M3 y M4 tienen mayor presencia que aquellos estructurados con los movimientos M1 y M5. 
Análisis de los resúmenes de artículos de investigación de dos áreas científicas: un estudio multidisciplinario

\begin{tabular}{|c|c|c|c|c|}
\hline \multirow{2}{*}{ Movimientos } & \multicolumn{2}{|c|}{$\begin{array}{c}\text { Ciencias } \\
\text { biomédicas }\end{array}$} & \multicolumn{2}{c|}{$\begin{array}{c}\text { Ciencias } \\
\text { sociales }\end{array}$} \\
\cline { 2 - 5 } & $\mathbf{F i}$ & $\mathbf{\%}$ & $\mathbf{F i}$ & $\mathbf{\%}$ \\
\hline 1 movimiento & 0 & 0.0 & 1 & 0,8 \\
\hline 2 movimientos & 4 & 2,6 & 20 & 16,7 \\
\hline 3 movimientos & 18 & 12.0 & 42 & 35,0 \\
\hline 4 movimientos & 121 & 80,7 & 42 & 35,0 \\
\hline 5 movimientos & 7 & 4,7 & 15 & 12,5 \\
\hline Total & $\mathbf{1 5 0}$ & $\mathbf{1 0 0}$ & $\mathbf{1 2 0}$ & $\mathbf{1 0 0}$ \\
\hline
\end{tabular}

Tabla 9. Relación porcentual del número de movimientos en los 270 resúmenes analizados en las revistas seleccionadas de las áreas de ciencias biomédicas y ciencias sociales

Otro dato que se debe resaltar es la relación entre el número de palabras del resumen y la información que contiene, expresada en movimientos. El promedio de palabras de los resúmenes de los artículos de investigación de las tres revistas de ciencias sociales analizadas es de 127 y el porcentaje promedio de cumplimiento de los movimientos es del 66 \%. Estos resultados difieren de los hallados en las revistas de ciencias biomédicas, en las que el promedio general de palabras de los resúmenes de los artículos de investigación es de 208,6 y el porcentaje promedio de movimientos es del 77 \%. Es importante resaltar que la cantidad recomendada para los resúmenes de artículos es de 150 a 250 palabras (APA, 2020; Day, 2005; Sánchez, 2016). En la Tabla 10 se puede comprobar esta afirmación.

\begin{tabular}{|c|c|c|c|}
\hline \multirow{2}{*}{ Área } & Revista & Palabras por resumen & $\begin{array}{c}\text { \% de } \\
\text { movimientos }\end{array}$ \\
\hline \multirow{4}{*}{$\begin{array}{c}\text { Ciencias } \\
\text { Sociales }\end{array}$} & Comunicación & 123,33 & 64 \\
\cline { 2 - 4 } & Psicología & 132,47 & 72 \\
\cline { 2 - 4 } & Lingüística & 128,03 & 63 \\
\cline { 2 - 4 } & Promedio general & $\mathbf{1 2 7 , 9 4}$ & $\mathbf{6 6}$ \\
\hline \multirow{4}{*}{$\begin{array}{c}\text { Ciencias } \\
\text { Biomédicas }\end{array}$} & Medicina humana I & 223,24 & 82 \\
\cline { 2 - 4 } & Medicina veterinaria & 171,96 & 70 \\
\cline { 2 - 4 } & Medicina humana II & 230,60 & $\mathbf{7 7}$ \\
\cline { 2 - 4 } & Promedio general & $\mathbf{2 0 8 , 6}$ & \\
\hline
\end{tabular}

Tabla 10. Relación porcentual entre el promedio de palabras por resumen y el porcentaje de movimientos

Al establecer las relaciones utilizando una correlación de Pearson — ver la Tabla 11—, el resultado es de 
Análisis de los resúmenes de artículos de investigación de dos áreas científicas: un estudio multidisciplinario 0,916, un índice alto y significativo, lo cual evidencia que, a mayor cantidad de palabras, mayor porcentaje promedio de presencia de movimientos.

\begin{tabular}{|c|c|c|}
\hline \multirow{2}{*}{} & Palabras \\
\hline \multirow{2}{*}{ Movimientos } & Sig. (bilateral) & $0,916^{*}$ \\
\cline { 2 - 3 } & $\mathbf{N}$ & 0,010 \\
\cline { 2 - 3 } & $\mathbf{N}$ & 6 \\
\hline
\end{tabular}

Tabla 11. Correlación de Pearson de las palabras empleadas en los resúmenes de las revistas seleccionadas de las áreas de ciencias biomédicas y ciencias sociales, donde la correlación es significativa en el nivel 0,05 bilateral

\section{Conclusiones}

En general, los resultados muestran que los movimientos 2 «¿De qué trata el estudio?»—, 3 «¿Cómo fue hecho?»- y 4 — ¿Qué fue descubierto?»— son de presencia obligatoria en las revistas de las tres disciplinas del área de ciencias sociales, mientras que los movimientos 1 —¿Qué sabemos acerca del tema?»-y 5 - «QQué significan los hallazgos?»_ tienen una presencia menor, aunque no menos significativa. Por otra parte, en las revistas del área de las ciencias biomédicas, los movimientos 2, 3, 4 y 5 son obligatorios. El movimiento 1 tiene una presencia menor en las revistas de medicina humana I y medicina veterinaria, y ninguna en la revista de medicina humana II. Estos resultados indican que existe una tendencia a resaltar los elementos estructurales de la investigación, descartando el aspecto contextual o explicativo del fenómeno, perteneciente al movimiento 1.

Frente a la organización retórica de los resúmenes estudiados se puede observar una alta variabilidad. En los resúmenes de las revistas de ciencias sociales predomina la estructura de 3 movimientos, pero en un orden diferente. Este resultado evidencia que en ellas el propósito de los autores no solo es contextualizar el fenómeno, sino reiterar determinada información, con el fin de proporcionar un mayor impacto discursivo en el lector. A su vez, los resúmenes del área de las ciencias biomédicas presentan una baja variabilidad, lo cual evidencia una estructura mucho más rígida que se acerca a la estructura del artículo de investigación. Los factores que explican estos fenómenos son variados, aunque resalta el hecho de que los editores de las revistas, al informar a los autores de los lineamientos para la publicación, no exponen las pautas para la redacción de este apartado, omisión que puede autorizar a los autores para estructurar el resumen según su criterio.

En lo que respecta al número de movimientos presentes en los resúmenes analizados, se detecta que solo en 7 
Análisis de los resúmenes de artículos de investigación de dos áreas científicas: un estudio multidisciplinario de los 150 resúmenes de las revistas del área ciencias biomédicas ocurren los cinco movimientos, mientras que en las revistas del área de ciencias sociales, 15 de los 120 resúmenes presentan los cinco movimientos. Ahora bien, la presencia de cuatro movimientos es mayor en las revistas del área de ciencias biomédicas con un 80,7\%, mientras que en las segundas los resúmenes con tres y cuatro movimientos comparten el mismo porcentaje, con un $35 \%$. Tales resultados muestran que la poca frecuencia de determinados movimientos - M1 y M5 para el caso de las revistas de ciencias sociales y M1 para el de las otras - no afecta el carácter informativo del resumen, aunque es importante destacar que el hecho de que las revistas de ciencias sociales presenten una mayor variabilidad en el orden y la cantidad de movimientos, les puede generar desventajas informativas frente a los resúmenes de las revistas del área de las ciencias biomédicas, lo que a la larga puede crear confusión o desinterés en el lector, quien no podrá hallar los datos necesarios para leer el artículo completo.

El promedio de las palabras de las tres revistas del área de ciencias sociales es de 127 y el porcentaje promedio de cumplimiento de los movimientos es del $66 \%$. Estos resultados difieren de los hallados en las revistas ciencias biomédicas, en las que el promedio general de palabras es de 208,6 y el porcentaje promedio de movimientos es del $77 \%$. Estos resultados muestran una mayor regularidad en los resúmenes de las revistas de ciencias biomédicas, pero, en líneas generales y al establecer las relaciones utilizando una correlación de Pearson, el resultado es de 0,916, un índice alto y significativo que demuestra que a mayor cantidad de palabras, mayor porcentaje promedio de presencia de movimientos.

Comunicar los resultados de una investigación es vital para el campo científico. Por eso, el resumen cumple una función importante dentro de esa difusión de la información, ya que es la carta de presentación de un AI. Por ello, conocer a fondo la forma en la que se organiza permite a los nuevos investigadores el perfeccionamiento de su redacción, logrando así un alto impacto en su formación profesional y académica. Los hallazgos de esta investigación tienen implicaciones pedagógicas, dado que esta apoya la generación de materiales que mejoren el proceso de enseñanza de la escritura académico-científica en las universidades, para finalmente fortalecer las capacidades discursivas de las comunidades que centran su quehacer en la creación de conocimiento. 
Análisis de los resúmenes de artículos de investigación de dos áreas científicas: un estudio multidisciplinario

\section{Referencias bibliográficas}

1. Alhuqbani, M. (2013). Genre-Based Analysis of Arabic Research Article Abstracts across Four Disciplines. Journal of Educational and Social Research, 3(3), 371-382.2. Altman, D. \& Gardner, M. (1987). More Informative Abstracts. Annals of Internal Medicine, 107(5), 790-791.

3. American Psychological Association. (2020). Publication Manual of the American Psychological Association (7th edition). American Psychological Association. https://doi.org/10.1037/0000165-000

4. Arias-Chávez, D., Ocaña-Fernández, Y. y Valenzuela-Fernández, A. (2019). Organización retórica del apartado del resumen de tesis peruanas de maestría en Educación. Revista EDUSER, 7(1), 1-17. https://doi.org/10.18050/ eduser.v7i1.2419

5. Atarama, T. \& Cortez, C. (2015). La gestión de la reputación digital en las universidades: Twitter como herramienta de la comunicación reputacional en las universidades peruanas. Revista de Comunicación, 14, 26-47. 6. Bhatia, V. (1993). Analyzing Genre: Language Use in Professional Settings. Longman.

7. Becerra, S. (2016). Descripción de las conductas de salud en un grupo de estudiantes universitarios de Lima. Revista de Psicología (PUCP),34(2), 239-260. https://dx.doi.org/10.18800/psico.201602.001

8. Beke, R. (2005). El metadiscurso interpersonal en artículos de investigación. Revista Signos, 38(57), 7-18. https://dx.doi.org/10.4067/S0718-09342005000100001

9. Can, S., Karabacak, E., \& Qin, J. (2016). Structure of Moves in Research Article Abstracts in Applied Linguistics. Publications, 4(23). 10.3390/publications4030023

10. Canale, G. y Coll, M. (2016). Historia y presente del yeísmo (rehilado) en el Uruguay. Lexis, 40(1), 5-40. http://revistas.pucp.edu.pe/index.php/lexis/article/view/15047

11. Cárcamo, B. (2020). La variación en los resúmenes de medicina y lingüística: un análisis desde la organización retórica y la teoría de la valoración. Onomáizen, Revista de Lingüística, Filología y Traducción, 47, 1-26. 10.7764/ onomazein.47.01

12. Charaudeau, P. (2012). Los géneros: una perspectiva socio-comunicativa. En Shiro, M.; Charaudeau, P. \& Granato, L. (Eds.), Los géneros discursivos desde múltiples perspectivas: teorías y análisis (pp. 19-44). Madrid: Iberoamericana, Vervuert.

13. Ciapuscio, G. (2005). La noción de género en la lingüística sistémico funcional y en la lingüística textual. Revista Signos, 38(57), 31-48. https://dx.doi.org/10.4067/S0718-09342005000100003

14. Cignetti, L. \& Guzmán, M. (2017). Análisis de la organización retórica de abstracts de artículos de investigación en medicina veterinaria. Texturas, 16, 41-57. 10.14409/texturas.v0i16.6938

15. Cross, C. \& Oppenheim, C. (2005). A Genre Analysis of Scientific Abstracts. Journal of Documentation, $62(4), 428-446$.

16. Dámaso-Mata, B., Chirinos-Cáceres, J. \& Menacho-Villafuerte, L. (2016). Estimación de costos económicos 
Análisis de los resúmenes de artículos de investigación de dos áreas científicas: un estudio multidisciplinario en la atención de la neumonía nosocomial en un hospital regional peruano, 2009 al 2011. Revista Peruana de Medicina Experimental y Salud Pública, 33(2), 233-240. https://doi.org/10.17843/rpmesp.2016.332.2202

17. Day, R. (2005). Cómo escribir y publicar trabajos científicos. Washington, D.C.: Organización Panamericana de la Salud.

18. Diez, M.B. (2007). El resumen de un artículo científico. Qué es y qué no es. Investigación y Educación en Enfermería, 25(1), 4-17.

19. Diez del Corral, A. (2018). Construcciones con «ser focalizador» en narcotelenovelas colombianas: de Antioquia al Valle del Cauca. Lexis, 42(1), 69-98. https://dx.doi.org/10.18800/lexis.201801.003

20. Donstrup, M. (2018). Vikings: poder e identidades culturales en la serie de History Channel. Revista de Comunicación, 17(1), 57-73. https://revistadecomunicacion.com/article/view/31

21. Dos Santos, M. (1996). The Textual Organization of Research Paper Abstracts in Applied Linguistics. Text, 16(4), 481-499.

22. El-Dakhs, D. (2018). Comparative Genre Analysis of Research Article Abstracts in More and Less Prestigious Journals: Linguistics Journals in Focus. Research in Language, 16(1), 47-63. 10.2478/rela-2018-0002

23. Espinosa, A., Freire, S. y Ferrándiz, J. (2016). Identificación colectiva y bienestar en una comunidad rural de la costa norte del Perú. Revista de Psicología, 34(1), 201-219. https://doi.org/10.18800/psico.201601.008

24. García-Cepero, M., Gómez-Hernández, F., Barrios-Martínez, D., Santamaría, A., Castro, L., Sánchez, A. y Zuluaga, Z. (2016). Itinerarios, hitos y catalizadores asociados a la emergencia del talento docente. Revista de Psicología (PUCP), 34(1), 85-115. https://dx.doi.org/10.18800/psico.201601.004

25. Gonzales-Achuy, E., Huamán-Espino, L., Aparco, J., Pillaca, J. y Gutiérrez, C. (2016). Factores asociados al cumplimiento del control de crecimiento y desarrollo del niño menor de un año en establecimientos de salud de Amazonas, Loreto y Pasco. Revista Peruana de Medicina Experimental y Salud Publica, 33(2), 224-232. https:// dx.doi.org/10.17843/rpmesp.2016.332.2187

26. Hartley, J. (1997). Is It Appropriate to Use Structured Abstracts in Social Science Journals? Learned Publishing, 10, 313-317. https://doi.org/10.1087/09531519750146789

27. Hartley, J. (2002). Do Structured Abstracts Take More Space? And Does It Matter? Journal of Information Science, 28(5), 417-422. https://doi.org/10.1177/016555150202800507

28. Hartley, J. \& Betts, L. (2009). Common Weaknesses in Traditional Abstracts in the Social Sciences. Journal of the American Society for Information Science and Technology, 60(10), 2010-2018. https://doi.org/10.1002/ asi.21102

29. Hyland, K. (2000). Disciplinary Discourses. Social Interactions in Academic Writing. London: Longman. 30. Hyland, K. (2005). Metadiscourse: Exploring Interaction in Writing. London: Continuum.31. Ibáñez, R. \& Moncada, F. (2017). El género resumen en dos disciplinas: una comparación a partir de su organización retórica y relaciones de coherencia. En R. Ibáñez y C. González (Eds.), Alfabetización disciplinar en la formación inicial docente: Leer y escribir para aprender (pp. 85-115). Valparaíso: Ediciones Universitarias de Valparaíso. 
Análisis de los resúmenes de artículos de investigación de dos áreas científicas: un estudio multidisciplinario 32. Khansari, D., Heng, Ch., Yuit, Ch. \& Tan, H. (2016). Regularities and Irregularities in Rhetorical Move Structure of Linguistics Abstracts in Research Articles. 3L: The Southeast Asian Journal of English Language Studies, 22(1), 39-54. http://doi.org/10.17576/3L-2016-2201-04

33. Lorés, R. (2004). On Research Article Abstracts: From Rhetorical Structure to Thematic Organization. English for Specific Purposes, 23(3), 280-302. http://dx.doi.org/10.1016/j.esp.2003.06.001

34. Lovón, M. (2019). Las palabras compuestas en el aimara de Conima. Lexis, 43(1), 55-85. https://dx.doi. org/10.18800/lexis.201901.002

35. Martín, P. (2003). Análisis contrastivo de los componentes estructurales y gramaticales de los resúmenes de los artículos científicos. Revista Española de Lingüística, 33(1), 153-183.

36. Martínez, G. (2016). Estructura sintáctica de las construcciones con verbos parasintéticos de los tipos a-...-ary en-...-ar. Lexis, 40(2), 243-292. http://revistas.pucp.edu.pe/index.php/lexis/article/view/16128

37. Mur, P. (2007). I/We Focus on...':A Cross-Cultural Analysis of Self-Mentions in Business Management Research Articles. Journal of English for Academic Purposes, 6(2), 143-162. https://doi.org/10.1016/j.jeap.2007.05.002

38. Piqué-Noguera, C. y Camaño-Puig, R. (2015). El resumen de artículo de investigación: análisis del género en un corpus de textos de enfermería. Signos, 48(87), 77-94. 10.4067/S0718-09342015000100004

39. Pho, P. (2008). Research Article Abstracts in Applied Linguistics and Educational Technology: A Study of Linguistic Realizations of Rhetorical Structure and Authorial Stance. Discourse Studies, 10(2), 231-250. https:// doi.org/10.1177/1461445607087010

40. Quintanilla,A. \& Kloss, E. (2019). Análisis de resúmenes: Una mirada para comprender la composición retórica de este género discursivo. Revista Espacios, 40(31). https://www.revistaespacios.com/a19v40n31/19403101.html 41. Sánchez, A. (2016). El género artículo científico: Escritura y análisis desde la alfabetización académica y la retórica funcional. Medellín: Editorial Fundación Universitaria Católica del Norte.42. Sabaj, O., Toro, P. \& Fuentes, M. (2011). Construcción de un modelo de movidas retóricas para el análisis de artículos de investigación del español. Onomázein, 24, 245-271.

43. Salager-Meyer, F. (2006). Medical Discourse: Structured Abstracts. In Brown, K. (Ed.), Encyclopedia of Language and Linguistics - 2nd Edition (pp. 717-720). Boston: Elsevier.44. Samraj, B. (2005). An Exploration of a Genre Set: RA Abstracts and Introductions in Two Disciplines. English for Specific Purposes, 24(2), $141-156$. 10.1016/j.esp.2002.10.001

45. Sánchez-Tito, M., \& Yañez-Chávez, E. (2015). Asociación entre el biotipo facial y la sobremordida: estudio piloto. Revista Estomatológica Herediana, 25(1), 5-11.46. Skelton, J. (1994). Analysis of Structure of Original Research Papers: An Aid to Writing Original Papers for Publication. British Journal of General Practice, 44, $455-$ 459. https://bjgp.org/content/bjgp/44/387/455.full. pdf

47. Suntara, W. \& Usaha, W. (2013). Research Article Abstracts in Two Related Disciplines: Rhetorical Variation between Linguistics and Applied Linguistics. English Language Teaching, 6(2), 84-99. 10.5539/elt.v6n2p84 48. Swales, J. M. (1990). Genre Analysis: English in Academic and Research Settings. Cambridge: Cambridge 
Análisis de los resúmenes de artículos de investigación de dos áreas científicas: un estudio multidisciplinario University Press.

49. Swales, J. M. (2004). Research Genres: Explorations and Applications. Cambridge: Cambridge University Press. https://doi.org/10.1017/CBO978113952482750. Swales, J. M. \& Feak, C. B. (2009). Abstracts and the Writing of Abstracts (Vol. 2). Ann Arbor: The University of Michigan Press. https://doi.org/10.3998/mpub.309332 51. Tseng, F. (2011). Analysis of Move Structure and Verb Tense of Research Article Abstracts in Applied Linguistics. International Journal of English Linguistics, 1(2), 27-35. 10.5539/ijel.v1n2p27

52. Weissberg, R. \& Buker, S. (1990). Writing up Research. Experimental Research Report Writing for Students of English. Englewood Cliffs, NJ: Prentice Hall Regents. 